\title{
Management of hydrocephalus in patients with tuberculous meningitis
}

\author{
Vedantam Rajshekhar \\ Department of Neurological Sciences, Christian Medical College, Vellore, India
}

Address for correspondence:

Dr. Vedantam Rajshekhar,

Department of Neurological

Sciences, Christian Medical College,

Vellore - 632 004, India.

E-mail: rajshekhar@cmcvellore.ac.in

DOI: $10.4103 / 0028-3886.55572$

\begin{abstract}
Hydrocephalus is one of the commonest complications of tuberculous meningitis (TBM) occurring in up to $85 \%$ of children with the disease. It is more severe in children than in adults. It could be either of the communicating type or the obstructive type with the former being more frequently seen. The Vellore grading system for clinical grading of patients with TBM and hydrocephalus with grade I being the best grade and grade IV being the worst grade has been validated by several authors. The management of hydrocephalus can include medical therapy with dehydrating agents and steroids for patients in good grades and those with communicating hydrocephalus. However, surgery is required for patients with obstructive hydrocephalus and those in poor grades. Surgery can involve either a ventriculo-peritoneal shunt or endoscopic third ventriculostomy (ETV). Complications of shunt surgery in patients with TBM and hydrocephalus are high with freeuent shunt obstructions and shunt infections reeuiring repeated revisions. ETV has variable success in these patients and is generally not advisable in patients in the acute stages of the disease. Mortality on long-term follow up has been reported to vary from $10.5 \%$ to $57.1 \%$ in those with altered sensorium prior to surgery and 0 to $12.5 \%$ in patients with normal sensorium. Surgery for patients in Vellore grade IV is usually associated with a poor outcome and high mortality and therefore, its utility in these patients is debatable
\end{abstract}

Key words: Brain, tuberculosis, hydrocephalus, grading, management, outcome

\section{Introduction}

Tuberculous meningitis (TBM) is still a very common problem in children and adults in India. Although there has been a reduction in the number of patients with TBM, it is estimated that in India the mortality due to TBM is approximately $1.5 / 100,000$ population. ${ }^{[1]}$ Hydrocephalus is one of the most common complications of TBM. It is almost always present in patients who have had the disease for four to six weeks. ${ }^{[2]}$ It is more frequent and severe in children than in adults and also occurs at an earlier stage in the disease process. ${ }^{[2]}$ The management of hydrocephalus in patients with TBM is discussed based on the available literature and an algorithm is suggested at the end of this review.

\section{Pathophysiology of Hydrocephalus}

Hydrocephalus in patients with TBM could be either of the communicating or the obstructive type, the former being more common. ${ }^{[3]}$ In both instances, the main cause is the inflammatory exudate occupying the subarachnoid spaces or the ventricular pathways. In the earlier stages of the disease, the thick gelatinous exudates block the subarachnoid spaces in the base of the brain (notably the interpeduncular and ambient cisterns) leading to communicating hydrocephalus. ${ }^{[4]}$ The exudates lead to a dense scarring of the subarachnoid spaces in the later stages of the disease again leading to communicating type of hydrocephalus. A communicating hydrocephalus can also result from the exudates blocking the arachnoid granulations which prevent the absorption of cerebrospinal fluid (CSF). The 
inflammation of the choroid plexus and ependyma also leads to an overproduction of CSF in the acute phase of the illness. This also contributes to the hydrocephalus and raised intracranial pressure. The obstructive type of hydrocephalus develops when the fourth ventricular outlets are blocked by the exudates or leptomeningeal scar tissue or when there is obstruction of the aqueduct either due to a strangulation of the brain stem by exudates or by a subependymal tuberculoma. Communicating type of hydrocephalus is more common in TBM than the obstructive type. Schoeman et al. ${ }^{[3]}$ found that the hydrocephalus was of the communicating type in $82 \%$ of their patients with TBM.

\section{Incidence of hydrocephalus}

Initial studies using air encephalography in patients with TBM found hydrocephalus in $62 \%$ of the patients ${ }^{[5]}$ Schoeman et al. ${ }^{[3]}$ found computer tomography (CT) evidence of hydrocephalus in $83 \%$ of 193 children with TBM. In a CT study, only three of 60 children and adults with TBM were found to have normal ventricles, giving an incidence of $95 \% .{ }^{[6]}$ Significantly, $87 \%$ of children in this study had severe hydrocephalus. Compared to an incidence of $71 \%$ in children, only $12 \%$ of adults with TBM had hydrocephalus. Thus, it is evident that hydrocephalus is more common in children with TBM compared to adults. Hydrocephalus is also more common in the later stages of the disease.

\section{Clinical Presentation}

The clinical features that suggest the presence of hydrocephalus are nonspecific. In any patient with TBM with altered sensorium, hydrocephalus should be suspected irrespective of the presence or absence of papilledema. Hydrocephalus is also likely to be present in patients who are alert and who complain of increasing headache with or without vomiting and blurring of vision.

\section{Grading of Patients}

Grading of patients with any disease is useful to prognosticate outcome. Although a widely used grading system exists for TBM, namely the MRC grading system [Table 1], a specific grading system did not exist for patients with TBM and hydrocephalus. We proposed a grading system (Vellore grading system) based on the presence or absence of neurological deficits and level of sensorium [Table 2]. ${ }^{[7]}$ This grading system is useful to grade the patients in retrospective studies but it has some degree of subjectivity in assessing sensorium. Therefore, the grading system was modified to include the Glasgow Coma Scale. ${ }^{[8]}$ The latter grading system is reproducible across different levels of clinical expertise and across different disciplines of healthcare workers and is, therefore, a more reliable system. Most importantly, both the grading systems have

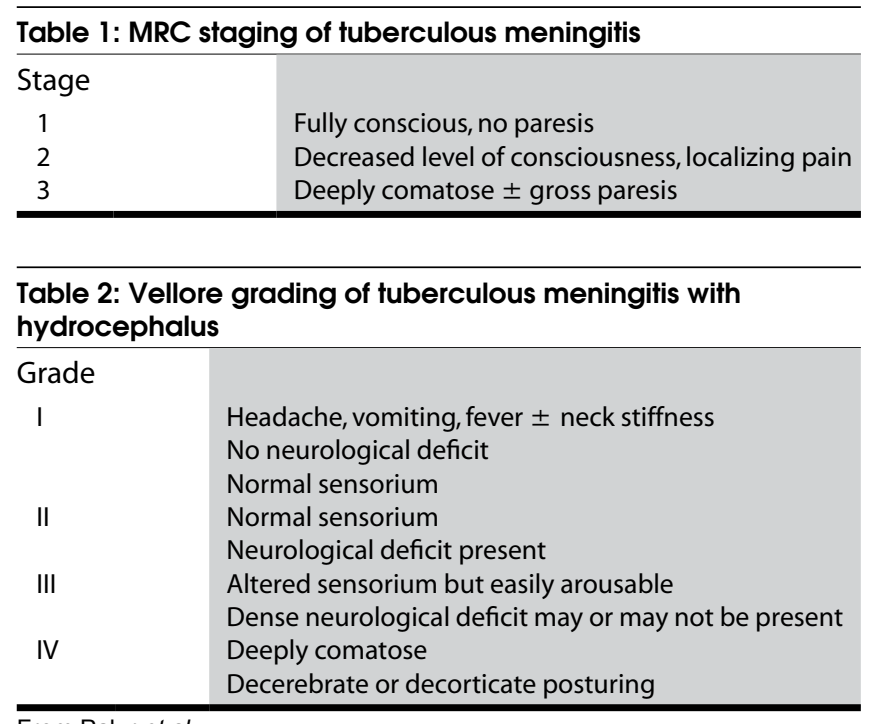

From Palur et al.

been shown to correlate with outcome and are hence valuable in prognosticating in the individual patients.

\section{Imaging}

A contrast CT scan is the imaging of choice in sick patients, especially children, as it can be performed without anesthesia or sedation and can be done in a short time compared to a magnetic resonance imaging (MRI) scan which might require a patient to still lie for longer periods. Adequate information regarding ventricular size, subependymal seepage, presence of infarcts and tuberculomas, and presence of basal exudates can be obtained from a contrast-enhanced CT scan. Although a CT scan might indicate whether the hydrocephalus is obstructive or communicating in nature, this differentiation is not always possible. CT is therefore not useful in determining the level of CSF block in TBM. Air-encephalography remains the most reliable way of determining the level of CSF obstruction. ${ }^{[9]}$ For example, in the cases where the CT scan shows dilatation of the lateral and third ventricles without a dilated fourth ventricle, it can safely be presumed that the hydrocephalus is due to obstruction to the CSF pathways in the region of the aqueduct. But if all the ventricles are dilated, then it is difficult to determine whether the hydrocephalus is due to obstruction of the fourth ventricular outlets or due to an obstruction to the CSF pathways in the basal subarachnoid spaces, unless the fourth ventricle is seen to be in communication with the cisterna magna. To make the differentiation between the two types of hydrocephalus, a dynamic study such as pneumoencephalogram or CT ventriculogram is needed. However, performing either of these investigations exposes the patient to an invasive procedure with attendant risks. 


\section{Medical Management}

Medical management might be offered as the first line of therapy to patients with communicating hydrocephalus. ${ }^{[10]}$ This comprises steroids, dehydrating agents such as mannitol, diuretics such as frusemide, and acetazolamide to reduce CSF production. Tapering doses of dexamethasone ( 12 to $16 \mathrm{mg} /$ day) can be given for four to six weeks. Mannitol can only be used for acute decompensation and is not recommended for more than 72 hours due to the occurrence of rebound intracranial hypertension. Acetazolamide $(100 \mathrm{mg} / \mathrm{kg})$ and frusemide $(1 \mathrm{mg} / \mathrm{kg})$ can be given for longer periods of up to a month. Medical management is generally successful in several patients with TBM who exhibit features of raised intracranial pressure. Along with the agents mentioned above, the antituberculous therapy (ATT) also probably helps in reducing the inflammatory response leading to opening of the CSF pathways. The addition of acetazolamide and furosemide was significantly more effective in achieving normal intracranial pressure (ICP) than antituberculous drugs alone. ${ }^{[3]}$ In a study from South Africa by Lamprect et al., ${ }^{[10]} 217$ children with MRC stage II and III TBM and hydrocephalus were initially managed medically if they had communicating hydrocephalus (as determined by pneumoencephalography or air encephalograms). This strategy allowed the authors to avoid shunt surgery in over $70 \%$ of the patients. Even in the other $30 \%$ who underwent shunt surgery, $41.5 \%$ (27 patients) had obstructive hydrocephalus. So it appears prudent to suggest medical management especially in patients with better sensorium (Vellore grade II) or if they definitely have communicating hydrocephalus. However, the patients should be monitored carefully for any worsening of sensorium or lack of improvement with medical management. In this situation a shunt might have to be done promptly.

Intrathecal hyaluronidase has been tried instead of shunt surgery in some patients with TBM and hydrocephalus and shown to have some benefit in the form of improvement in sensorium. But it did not offer any advantage over shunt insertion and this therapy is presently not commonly practised..$^{[3,11]}$

\section{Surgical management}

Early attempts at surgical management of hydrocephalus in patients with TBM included repeated tapping of the ventricles through burr holes, suboccipital decompression, lateral and third ventriculostomy (open) and ventriculo-subarachnoid shunts. ${ }^{[12]}$ The availability of reliable shunt systems in the early 1960s dramatically changed the management of hydrocephalus.

\section{Indications for surgery}

Patients in grades I and II should undergo surgery at the earliest. It has been shown that outcomes are better with early surgery especially in patients in the better grades. The South African group has suggested that early surgery be reserved for those with obstructive hydrocephalus and medical therapy be tried for those with communicating hydrocephalus. ${ }^{[10]}$ However, one of the risks of such a strategy is that some patients undergoing medical therapy might deteriorate rapidly and have a poor outcome if shunt surgery is delayed. Medical therapy also implies continuous monitoring of the patient in the hospital for prolonged periods which might not be cost-effective.

Not all patients who undergo shunt surgery for TBM with hydrocephalus show a significant improvement in their sensorium or symptoms. It is, therefore, evident that the cause of the altered sensorium and other symptoms in these patients are not always or completely attributable to the hydrocephalus. The encephalitis that is caused by the inflammatory processes enveloping the cerebral hemispheres, the ischemia caused by the arteritis especially of the small perforators at the base of the brain probably contribute in equal if not more than equal measure to the altered sensorium. A considerable proportion of patients with TBM especially those in the poorer grades have been shown to have infarcts of the basal ganglia, thalamus and brain stem. It is the same group of patients who will also exhibit hydrocephalus on the CT scans. Thus, in grade III and IV patients, it becomes difficult to determine the cause of the alteration of sensorium.

\section{Shunt surgery}

Ventriculo-atrial (VA) shunts were the initial choice for shunt procedures. The fear of disseminating the tuberculous disease through such shunt system was dispelled by reports by Bhagwati and others, ${ }^{[13-16]}$ who described the successful use of VA shunts in patients with TBM. However, others have reported occasional cases of dissemination of the disease following a VA shunt. ${ }^{[17]}$ There was a shift to ventriculo-peritoneal (VP) shunt in the early 1980s and since then VP shunt has remained the shunt procedure of choice for patients with TBM and hydrocephalus. Although, there is a theoretical risk of developing tuberculous peritonitis following a VP shunt, to the best of our knowledge such an occurrence has not been reported. The technique for shunt surgery does not differ from that used for hydrocephalus from any other cause.

\section{Endoscopic third ventriculostomy}

Since the popularization of endoscopic third ventriculostomy (ETV) in the mid 1990s, there has been an interest in avoiding shunts in patients with hydrocephalus with the hope that if a foreign body insertion could be 
avoided, the accompanying complications of a shunt (infection, blockage, extrusion, abdominal pseudocysts, skin erosion over shunt components) could also be avoided. Initially, Figaji et al. ${ }^{[18]}$ and we described the use of ETV in TBM related hydrocephalus in two patients each. ${ }^{[19]}$ Since then, there have been several reports on the use of ETV in these patients with varying degrees of success.

Singh et al. ${ }^{[20]}$ reported a success rate of ETV in $77 \%$ of 35 patients with TBM and hydrocephalus. Also, $60 \%$ had early and $17 \%$ had delayed recovery. The success rates were not related to the type of hydrocephalus (obstructive or communicating). However, the presence of a thin and transparent floor of the third ventricle seemed to be associated with a higher success rate of $87 \%$. Hussain et al. ${ }^{[21]}$ determined that $68 \%$ of the patients with TBM and hydrocephalus benefited from various endoscopic procedures including ETV. In a more recent study by the same group they found that 19 of 26 (73\%) patients with TBM and hydrocephalus responded to ETV. ${ }^{[22]}$ They found that patients with longer duration of symptoms and ATT were more likely to benefit from the ETV. In contrast, Figaji et al. ${ }^{[23]}$ reported a successful outcome following attempted ETV in only seven of $17(41 \%)$ patients with TBM and hydrocephalus. In five patients, the ETV was performed but failed, whereas in another five the procedure had to be abandoned due to abnormal anatomy. They concluded that although ETV was technically possible in patients with TBM, only a surgeon experienced in endoscopic procedures should perform the surgery as the procedure is more demanding than in other situations.

In patients with TBM, the floor of the third ventricle is frequently thick and the subarachnoid space is also likely to be obliterated by exudates making it difficult to recognise anatomical landmarks. In this situation it might be prudent to abandon the procedure than risk injury to the basilar artery and its branches. We have attempted ETV in patients with TBM early in the course of disease and found that the floor of the third ventricle besides being thick is coated with small tubercles and granulation tissue that bleed when touched with even a blunt probe. As bleeding of even minor intensity can obscure the field during endoscopic procedures, we believe that ETV might be better avoided for acute hydrocephalus in patients with TBM and be reserved for either in those who have been treated with ATT for at least four weeks or in whom the disease is burnt out and the hydrocephalus has developed late. Chugh et al. ${ }^{[2]}$ found that the outcome was better in those who received ATT for four weeks prior to ETV than in those operated earlier. It could also be considered in patients who have shunt failure, as it might be a better option than shunt revision in these patients.

\section{Complications of surgery}

\section{Shunt surgery}

It has been reported that complications of shunt surgery are higher in patients with TBM than in patients with other conditions. The reasons for this are the poor general condition of these patients and also the presence of higher protein and cellular content in the CSF leading to more frequent shunt obstruction. Agarwal et al. ${ }^{[24]}$ reported shunt related complications in $11(30 \%)$ children and three of 37 children had to undergo multiple shunt revisions. Palur et al. ${ }^{[7]}$ reported that 26 of 114 (22.8\%) patients had to undergo one or more shunt revisions, one patient requiring more than three revisions. Sil and Chatterjee ${ }^{[25]}$ reported a shunt infection rate of $15.6 \%$ and revision rate of $43.8 \%$ in their series of 37 children who underwent shunt surgery for TBM with hydrocephalus. Multiple revisions were done in $18.7 \%$ of patients.

Shunt infection and erosion of skin over the shunt components are the other major complications of shunt surgery in poor grade patients with TBM and hydrocephalus.

\section{Endoscopic third ventriculostomy}

Failure of the ETV with CSF leak and bleeding during the surgery are the most commonly reported complications of ETV. Husain et al..$^{[21]}$ had complications in three of 28 patients undergoing ETV for TBM related hydrocephalus. In a more recent series, the same authors have reported ETV failures in seven of 26 patients. ${ }^{[22]}$ They did not report any other complications in this series of patients. Risk of injury to the basilar artery and its branches is theoretically higher in patients with TBM due to the thickened and opaque third ventricular floor.

\section{Outcome}

All the early reports of shunt surgery for hydrocephalus associated with TBM report poor outcome in a proportion of patients. Bhagwati ${ }^{[13]}$ reported mortality in 3 of 7 patients undergoing shunt surgery. Only 4 of 9 patients treated with the Updhyaya VA shunt improved following surgery. ${ }^{[15]}$ The other 5 patients died at varying periods of time after the surgery. Palur et al..$^{[9]}$ reported that of the 114 patients followed up for an average of 45.6 months following shunt surgery for TBM and hydrocephalus, $48(42.1 \%)$ died. 63 (55\%) had a good outcome or moderate disability. Agarwal et al. ${ }^{[21]}$ reported that 29 of 37 children $(78 \%)$ had a good outcome or moderate disability at 3 months follow-up following shunt surgery for TBM and hydrocephalus. In various series of shunt surgery for TBM with hydrocephalus reported up to 1991, the mortality rate for those with altered sensorium has ranged from $10.5 \%$ to $57.1 \%$ and for those with normal sensorium has ranged from 0 to $12.5 \% \cdot{ }^{[7,14,26,27]}$ The mortality rates might 
be higher if the follow-up duration is longer as those with severe disability might go on to die. This is borne out by the fact that in our initial retrospective study, 14 of the $48(29.1 \%)$ patients who died, did so more than one year after the surgery. ${ }^{[7]}$ Therefore, while considering outcome patients discharged with severe disability should be categorized with those who have died.

\section{Prognostic factors}

In our series of patients, 114 patients who were followed up for 6 months to 13 years (mean 45.6 months) following shunt surgery for TBM with hydrocephalus, we studied various prognostic factors to predict outcome following shunt surgery. ${ }^{[7]}$ Age $(0-9,10+$ years), duration of altered sensorium ( $<15$ days, $>15$ days), CSF cell count $(<50,>50 / \mathrm{ml})$, CSF protein level $(<50,>50 \mathrm{mg} \%)$, shunt revisions $(0,1+)$ and bilateral shunts $(0,1)$, did not have any signficant effect on the long term outcome. Only the admission grade [Table 2] was highly significant $(P=0.0001)$ in predicting the outcome. The presence of infarcts in the basal ganglia and internal capsule are also likely to indicate a poor outcome following shunting.

It is clear from several subsequent studies that the grade at presentation is the best and most consistent predictor of outcome following shunt surgery in patients with TBM. Agarwal et al. ${ }^{[24]}$ studied 37 children with TBM and hydrocephalus who had undergone shunt surgery. They used the Vellore grading system proposed by us [Table 2] and found that all patients in grade IV were left with severe disability or died. They recommended shunt placement in all children of grade II and III as this policy has yielded the best results. For grade IV children external ventricular drainage, followed by shunting if improvement occurs remains the most cost-effective procedure.

\begin{tabular}{ll}
\hline $\begin{array}{l}\text { Table 3: Modified Vellore grading of tuberculous meningitis } \\
\text { and hydrocephalus }\end{array}$ \\
$\begin{array}{ll}\text { Grade } \\
\text { I }\end{array}$ \\
$\begin{array}{ll}\text { GCS 15 } \\
\text { Headache, vomiting, fever } \pm \text { neck stiffness } \\
\text { No neurological deficit } \\
\text { GCS 15 } \\
\text { III } & \text { Neurological deficit present } \\
\text { GCS 9-14 } \\
\text { IV }\end{array}$ \\
$\begin{array}{l}\text { Neurological deficit may or may not be present } \\
\text { GCS 3-8 }\end{array}$ \\
\hline
\end{tabular}

From Mathew et al. GCS - Glasgow coma score
Singh and Kumar ${ }^{[28]}$ reported the outcome of VP shunt in 140 children who underwent shunt surgery for TBM and hydrocephalus. They found a good correlation between the Vellore grade at shunt surgery with the outcome. All the mortalities occurred in patients in grade IV (43/87).

In a follow-up prospective study, ${ }^{[8]}$ we validated the utility of our grading system. We used an algorithm proposed in our initial article in 1991 and evaluated 32 consecutive patients with TBM and hydrocephalus in grades III or IV. We used external ventricular drainage (EVD) to select patients for VP shunt. All but one of the patients in grade IV did not improve with EVD and succumbed to the disease.

The grading system proposed by us and modified to include the GCS [Table 3] is a valid system to predict outcome following shunt surgery in patients with TBM and hydrocephalus as has been verified by several studies. Table 4 shows the outcome in patients who underwent shunt surgery categorized using our grading system.

Sil and Chatterjee ${ }^{[25]}$ noted that all patients who fared poorly following shunt surgery, in their series of 37 children with TBM and hydrocephalus, had evidence of infarcts in their CT scans. It is also likely that these patients were in the poorer Vellore grades before shunting. Stated otherwise, those in poorer grades are likely to have infarcts and therefore, infarcts on CT scan might not be an independent predictor of outcome.

Kemaloglu et al. ${ }^{[29]}$ noted that patients with TBM with mild and moderate hydrocephalus who underwent early shunt surgery (two days after diagnosis) had better outcomes compared to those who had delayed surgery (three weeks after diagnosis). This effect was not seen in patients with severe hydrocephalus. While this suggests that early surgery should be offered to patients with mild and moderate hydrocephalus without delaying it while waiting to determine the effect of medical therapy, it is unclear whether shunt surgery could have been avoided in some of these patients.

Table 4: Preoperative Vellore grade versus poor outcome following shunt surgery*

\begin{tabular}{lcccccc}
\hline Authors (year) & No. of patients & \multicolumn{3}{c}{ Preoperative Vellore grade } & Follow-up (months) \\
\cline { 3 - 6 } & & I & II & III & IV & \\
\hline Palur et al. (1991) & 114 & 20 & 34.7 & 51.9 & 100 & 45.6 \\
Singh and Kumar (1996) & 140 & 0 & 0 & 39.7 & 65.5 & $\mathrm{NA}^{\#}$ \\
Mathew et al. (1998) & 32 & - & - & 21.8 & 91.7 & 23.1 \\
Agarwal et al.** (2005) & 37 & - & 38 & 60 & 100 & 9 \\
Sil and Chatterjee (2008) & 37 & & - & & - & $6-12$ \\
\hline
\end{tabular}

*\% of patients who died or had severe disability; ${ }^{\text {no }}$ data; **there were no patients in grade I; - no patients in that grade; and included grades II and III patients together 


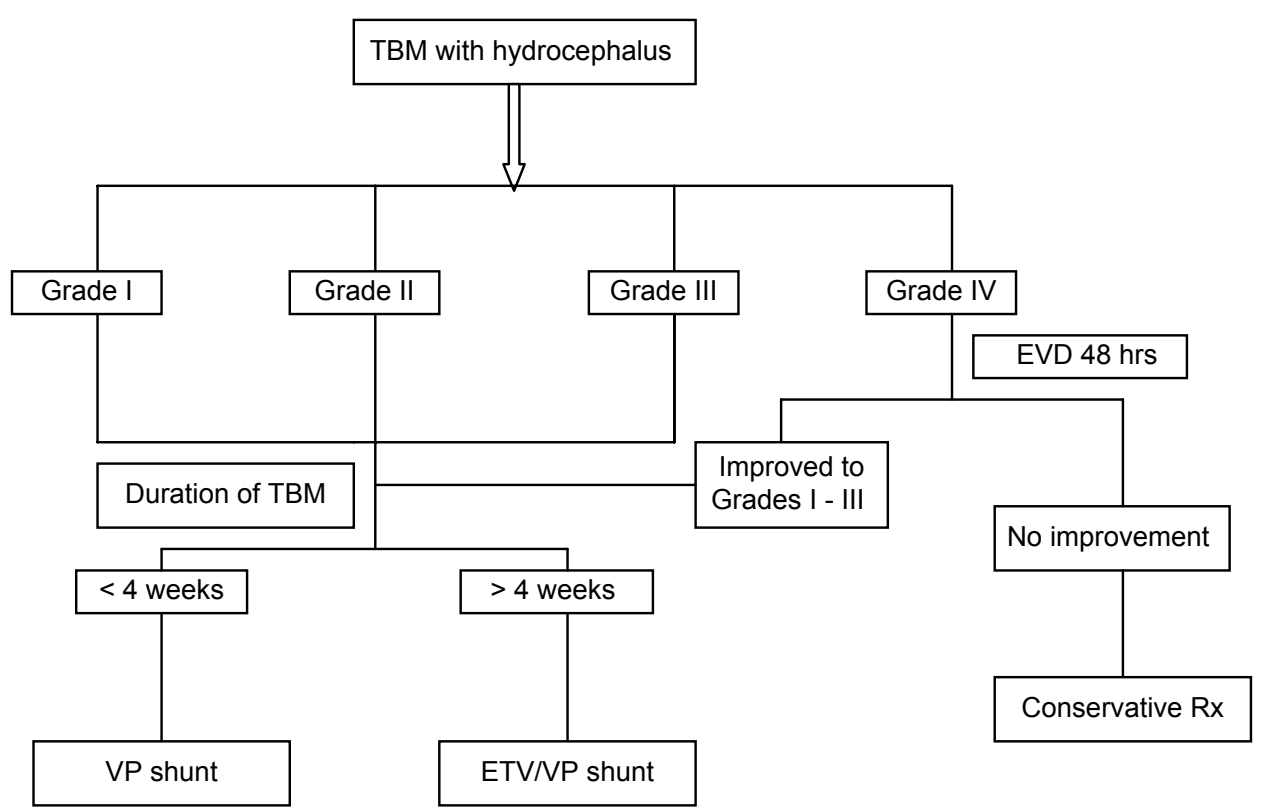

Figure 1: Suggested algorithm for management of TBM with hydrocephalus

TBM in HIV-positive patients and drug resistant tuberculous In a comparative study of 30 patients with TBM and hydrocephalus, 15 being HIV-positive, it was found that mortality rate and poor outcomes at one month following shunt surgery and ATT, were considerably higher in HIV-positive patients compared to those who were HIV-negative. ${ }^{[30]}$ The HIV-positive group had a mortality rate of $66.7 \%$ and poor outcome in $64.7 \%$ compared with mortality rate of $26.7 \%$ and poor outcome in $30.8 \%$ in the HIV-negative group. No patient in the HIV-positive group experienced a good recovery. No patient (either HIVpositive or HIV-negative) who presented in TBM Grade 4 survived, whereas no HIV-positive patient who presented in TBM Grade 3 survived. A significant relationship was noted between CD4 counts and patient outcomes $(P<0.031)$. It was suggested that HIV-positive patients with TBM should undergo a trial of ventricular or lumbar CSF drainage, and only those who show an improvement following EVD should undergo shunt surgery.

The outcome of TBM with hydrocephalus is finally dependent on the response of the disease to ATT. It is understandable that in patients with drug resistant $\mathrm{TB}$ either multi-drug resistant TB (MDR-TB) or extensively drug resistant TB (XDR-TB), the outcome is likely to be poor. There are however, no studies which have reported on the outcome following surgery for drug resistant TBM.

\section{Suggested management algorithm}

The main aim of the suggested algorithm is to improve the outcome of patients with TBM and hydrocephalus and also to avoid shunt surgery in those who are unlikely to benefit from the surgery [Figure 1]. The grading of the patient is important in deciding on further course of action. Although medical management could be tried for a few days or a week in patients in grades I and II, the patient should be monitored closely during this period to detect any worsening or lack of improvement and a shunt should be promptly offered in case of failure of medical management. Prolonging medical therapy in patients in good grades could be harmful and may lead to irreversible brain damage.

\section{References}

1. Chakraborty AK. Estimating mortality from tubereulous meningitis in a community: Use of available epidemiological parameters in the Indian context. Indian J Tubere 2000;47:9-13.

2. Tandon PN. Tuberculous meningitis (cranial and spinal). In: Vinken PJ, Bruyn GW, editors. Handbook of Clinical Neurology. Infections of the Nervous System. vol. 33. Amsterdam: North—Holland; 1978. p. 195-262.

3. Schoeman J, Donald P, van Zyl L, Keet M, Wait J. Tuberculous hydrocephalus: Comparison of different treatments with regard to ICP, ventricular size and clinical outcome. Dev Med Child Neurol 1991;33:396-405.

4. Dastur DK, Manghani DK, Udani PM. Pathology and pathogenetic mechanisms in neurotuberculosis. Radiol Clin North Am 1995;33:733-52.

5. Lorber J. Studies of CSF circulation in tuberculous meningitis. II. A review of 100 pneumoencephalograms. Arch Dis Child 1951;26:28-48.

6. Bhargava S, Gupta AK, Tandon PN. Tuberculous meningitis-A CT scan study. Br J Radiol 1982;55:189-96.

7. Palur R, Rajshekhar V, Chandy MJ, Joseph T, Abraham J. Shunt surgery for hydrocephalous in tubercular meningitis: A long-term follow-up study. J Neurosurg 1991;74:64-9.

8. Mathew JM, Rajshekhar V, Chandy MJ. Shunt surgery for poor grade patients with tuberculous meningitis and hydrocephalus: Effect of response to external ventricular drainage and other factors on long-term outcome. J Neurol Neurosurg Psychiatry 1998;65:115-8.

9. Bruwer GE, Van der Westhuizen S, Lombard CJ, Schoeman JF. Can CT predict the level of CSF block in tuberculous hydrocephalus? Childs Nerv Syst 2004;20:183-7.

10. Lamprecht D, Schoeman J, Donald P, Hartzenberg H. Ventriculoperitoneal shunting in childhood tuberculous meningitis. Br J Neurosurg 2001;15:119-25. 
11. Bhagwati SN, George K. Use of intrathecal hyaluronidase in the management of tuberculous meningitis with hydrocephalus. Childs Nerv Syst 1986;2:20-5.

12. Cairns H. Neurosurgical methods in the treatment of tuberculous meningitis. Arch Dis Child 1951;26:376-83.

13. Bhagwati SN. Ventriculoatrial shunt in tubercular meningitis with hydrocephalus. J Neurosurg 1971;35:309-13.

14. Roy TK, Sircar PK, Chandar V. Ventriculoatrial shunt in the management of tuberculous meningitis. Indian Pediatr 1979;16:1023-7.

15. Chitale VR, Kasaliwal GT. Our experience of ventriculoatrial shunt using Upadhyaya valve in cases of hydrocephalus associated with tuberculous meningitis. Prog Pediatr Surg 1982;15:223-36.

16. Upadhyaya P, Bhargava S, Sundaram KR, Mitra DK, George J, Singh DC. Hydrocephalus caused by tuberculous meningitis: Clinical picture, CT findings and results of shunt surgery. Z Kinderchir 1983;38:76-9.

17. Murray HW, Bandstetter RD, Levyne MH. Ventriculoatrial shunt for hydrocephalus complicating tuberculous meningitis. Am J Med 1981;70:895-8

18. Figaji AA, Fieggen AG, Peter JC. Endoscopic third ventriculostomy in tuberculous meningitis. Childs Nerv Syst 2003;19:217-25.

19. Jonathan A, Rajshekhar V. Endoscopic third ventriculostomy for chronic hydrocephalus following tuberculous meningitis. Surgical Neurology 2005;63:32-4.

20. Singh D, Sachdev V, Singh AK, Sinha S. Endoscopic third ventriculostomy in post-tubercular meningitic hydrocephalus: A preliminary report. Minim Invasive Neurosurg 2005;48:47-52.

21. Husain M, Jha DK, Rastogi M, Husain N, Gupta RK. Role of neuroendoscopy in the management of patients with tuberculous meningitis hydrocephalus. Neurosurg Rev 2005;28:278-83.

22. Chugh A, Husain M, Gupta RK, Ojha BK, Chandra A, Rastogi M. Surgical outcome of tuberculous meningitis hydrocephalus treated by endoscopic third ventriculostomy: Prognostic factors and postoperative neuroimaging for functional assessment of ventriculostomy. J Neurosurg Pediatr 2009;3:371-7.

23. Figaji AA, Fieggen AG, Peter JC. Endoscopy for tuberculous hydrocephalus. Childs Nerv Syst 2007;23:79-84.

24. Agrawal D, Gupta A, Mehta VS. Role of shunt surgery in pediatric tubercular meningitis with hydrocephalus. Indian Pediatr 2005;42:245-50.

25. Sil K, Chatterjee S. Shunting in tuberculous meningitis: A neurosurgeon's nightmare. Childs Nerv Syst 2008;24:1029-32.

26. Bullock MR, Van Dellen JR. The role of cerebrospinal fluid shunting in tuberculous meningitis. Surg Neurol 1982;18:274-7.

27. Gelabert M, Castro-Gago M. Hydrocephalus and tubercular meningitis in children. Child's Nerv Syst 1988;4:268-70.

28. Singh D, Kumar S. Ventriculoperitoneal shunt in post tubercular hydrocephalus. Ind Pediatr 1996;33:854-5.

29. Kemaloglu S, Ozkan U, Bukte Y, Ceviz A, Ozates M. Timing of shunt surgery in childhood tuberculous meningitis with hydrocephalus. Pediatr Neurosurg 2002;37:194-8.

30. Nadvi SS, Nathoo N, Annamalai K, van Dellen JR, Bhigjee AI. Role of cerebrospinal fluid shunting for human immunodeficiency virus-positive patients with tuberculous meningitis and hydrocephalus. Neurosurgery 2000;47:644-9

Accepted on 02-07-2009

Source of Support: Nil, Conflict of Interest: None declared. 